\title{
Coping Strategies of Mothers of Children with Cardiac Problems
}

\author{
Gehan M. Mounir*, Mostafa A. Abolfotouh*, Kholoud Y. Tayel*
}

\begin{abstract}
Background: Assessment of coping strategies is important in planning of health education programs to enhance coping. Objective: The aim of the present work was to assess the knowledge and attitude of mothers concerning cardiac problems, to assess the coping strategies of mothers of children with cardiac problems, and to identify some predictors of the coping strategies. Methods: A cross sectional study of 187 mothers of children with cardiac problems who attended the cardiology school health insurance clinic of Sporting Student's Hospital in Alexandria with their children within a period of one month was conducted. Every mother was subjected to the modified Jalowiec coping scale to assess the coping strategies. Knowledge and attitude of the mothers were assessed through a pre-designed interviewing questionnaire. Results: The results showed marked variability in correct responses to various statements about cardiac problems. However, most of mothers showed poor knowledge $(87.17 \%)$ and about half of them (46.0\%) showed negative attitude. These figures were significantly higher among mothers of children with congenital heart defects (CHD) than among those whose children suffer from rheumatic heart disease (RHD). The most commonly used coping method was "Pray; trust in god" (92.5\%), followed by "Accept the situation as it is" (88.0\%), and "Hope that things will be better" $(73.5 \%)$. More than half of the mothers (55.5\%) reported that they always "Resign themselves to the situation because it is their fate" while $28.0 \%$ reported that they always "Worry." The percent mean score of use of problem-oriented coping methods was nearly equal to that for affective-oriented coping methods. Duration of illness $(p<0.01)$, previous hospitalization $(p=0.01)$, and attitude of mothers to illness $(p=0.05)$ were all significantly associated with coping strategies. However, after adjustment for other variables, high percent mean total coping scores were more likely among mothers of children with longer disease duration and those whose mother showed negative attitude towards cardiac problems $(p<0.05)$. Conclusion: Health education programs are needed to enhance coping strategies, knowledge, and attitude of mothers of children with cardiac problems. However, high coping strategy scores are significantly associated with the longer duration of illness and the negative attitude of mothers, possibly due to prolonged exposure to stress and the feeling that their children are different. Support group for parents is a highly recommended strategy to help a family benefit from communication with others who share common concerns, interests, and goals.
\end{abstract}

\section{INTRODUCTION}

Rheumatic and congenital heart diseases are still significant causes of morbidity and mortality in Egyptian children. ${ }^{1}$ In Egypt, rheumatic heart disease (RHD) is a significant health problem, with an estimated prevalence rate of $5.1 / 1000$ school children. ${ }^{2}$ On the other hand, there is little information on the prevalence of congenital heart disease ( $\mathrm{CHD}$ ) in school-age children in Egypt. It is estimated that 8 of every 1,000 babies are born with a congenital heart defect. ${ }^{3}$

With improvements in longevity and quality of life, these children and adolescents, and their families have become the focus of scientific investigation. ${ }^{4}$ As a result of these advances, caregivers of these children are subject to many potential stressors including the scheduling of frequent clinic visits,

*Family Health Department (School Health) High Institute of Public Health, Alexandria University, Egypt. 
monitoring and limiting their child's activities, and administering medication. The findings of a previous study upon school children with cardiac problems in Alexandria showed nonparticipation in physical education lessons of $72.2 \%$ and $80 \%$ of $\mathrm{RHD}$ and $\mathrm{CHD}$ children respectively, mainly due to private doctor's certificate for non-participation and parental overprotection. ${ }^{5}$ Thus, they must adapt to novel responsibilities in the care of their children as well as endure continued psychological and financial stressors associated with having a child with a potentially life-threatening chronic illness. ${ }^{3}$

As the family's stress increases, the likelihood of treatment compliance for the ill child decreases. For some illnesses, psychological stress directly affects the physiological processes that regulate symptom expression. Thus, the potential for a vicious circle is created as coping with a child's illness heightens family stress, which in turn reduces treatment compliance and increases the occurrence of symptoms, which may then further heighten family stress, and so on. 6
Parental coping is a major aspect of children coping with chronic diseases. The manner in which parents cope can affect family functioning, the child's functioning and most importantly, how successful the child's needs are being met. ${ }^{7}$ Coping strategies refer to the specific efforts, both behavioral and psychological, that people employ to master, tolerate, reduce, or minimize stressful events. Two general coping strategies have been distinguished: problem-solving strategies are efforts to do something active to alleviate stressful circumstances, whereas emotionfocused coping strategies involve efforts to regulate the emotional consequences of stressful or potentially stressful events. ${ }^{8}$

Research indicates that people use both types of strategies to combat most stressful events. The predominance of one type of strategy over another is determined, in part, by personal style (e.g., some people cope more actively than others) and also by the type of stressful event; for example, people typically employ problem-focused coping to deal with potential controllable problems such as work- 
related problems and family-related problems, whereas stressors perceived as less controllable, such as certain kinds of physical health problems, prompt more emotionfocused coping. ${ }^{9}$

Assessment of coping strategies is important in planning of health education programs to enhance coping. Unfortunately, such work on mothers of children with cardiac problems has lagged far behind. The aim of the present work was: 1) to assess the knowledge and attitude of mothers concerning cardiac problems, 2) to assess the coping strategies of mothers of children with cardiac problems, and 3) to identify some predictors of the coping strategies.

\section{MATERIAL AND METHODS}

\section{Study setting and sampling:}

The study was conducted at the Cardiology Clinic of Sporting Student's Hospital in Alexandria which is affiliated to the Health Insurance Organization. All mothers of children with cardiac problems who attended this clinic with their children during March 2007 and accepted to participate in the study were subjected to a cross sectional study. The total sample was 220 mothers. Those who responded were 187 mothers making a response rate of $85 \%$

\section{Methods:}

For the execution of the present study, the following tools were used:

1) Modified Jalowiec coping scale (JCS) ${ }^{10}$ was used to assess the coping strategies of the mothers. Translation into Arabic version was performed by Kanona $(2002)^{11}$. This scale contained 21 items. These items were scored on 3 point Likert scale to indicate extent of use; no (0), sometimes (1), and always (2). Higher scores indicate better coping. These items were divided into two groups: the first group is the affective-oriented coping strategies including 14 statements with a total score ranging from 0 to 28 points. The second group is the problem-oriented coping strategies including 7 statements with a total score ranging from 0 to 14 points. For each mother, the total score was calculated together with the affective- 
oriented and problem-oriented scores.

The percent mean score (and standard deviation) was calculated.

2) $\boldsymbol{A}$ pre-designed interviewing questionnaire was used to collect the following data from the mothers:

a) Personal data: including age, sex, and birth order of the child. Socioeconomic data including mother's education, mother's work, and family size were also collected.

b) Scholastic achievement: the results of the previous academic year for each student were recorded.

c) Disease characteristics: including child diagnosis (congenital or rheumatic), type of cardiac lesion, age at diagnosis, duration of illness, and previous hospitalization.

d) Mother's reaction after diagnosis: such as shock, sadness, deny, or acceptance.

e) Knowledge statements: in the form of 6 items with multiple choice answers about mother's knowledge of the child diagnosis, cause, prevention, treatment, and penicillin prophylaxis (frequency and duration). The total knowledge score ranged from 0 to 14 points. Those with scores 8 or more ( $\geq 60 \%$ of total score) were considered of fair/good knowledge. Those with score less than $8(<60 \%$ of total score) were considered of poor knowledge.

f) Attitude statements: in the form of 9 items measuring mother's attitude towards their diseased child. Each item consisted of two choices; agree (0) and do not agree (1). Positive attitude scored (1) and negative attitude scored (0). The total score ranged from 0 to 9 points. Those with scores 5 or more ( $\geq$ $60 \%$ of total score) were considered having positive attitude. Those with scores less than $5(<60 \%$ of total score $)$ were considered having negative attitude.

\section{Statistical analysis:}

The data were coded, entered, and analyzed using SPSS (version 10) software 
program. Mean and standard deviation, Chisquared test, ANOVA, Mann-Whitney test, and multiple regression analysis were applied. The p-value of $<0.05$ was considered significant.

\section{RESULTS}

Table 1 shows that the age of the children ranged from one month to 18 years with a mean age of $5.47 \pm 5.21$ years. The age at diagnosis ranged from birth to 15 years with a median age at diagnosis of 0.7 years. Duration of illness ranged from one month to 17 years with a mean duration of illness $2.55 \pm 2.90$ years. Males constituted $57.8 \%$ of the sample and $42.5 \%$ were females. Concerning mother education, $92.5 \%$ were of low and middle education and only $7.5 \%$ were of high education. Most of the mothers were not working (90.4\%) and only 9.6\% were working.

As regards cardiac lesions, about three quarter of the sample $(75.4 \%)$ reported single lesion, which was significantly higher among the congenital cases (81.8\%), compared to $\mathrm{RF} / \mathrm{RHD}$ cases $(51.3 \%)$. On the other hand, multiple lesions were significantly higher among RF/RHD cases (25.6\%) as compared to the congenital cases $(18.2 \%)\left(\chi^{2}=38.81, p<\right.$ 0.00). Previous hospitalization was reported by $42.8 \%$ of mothers.

Concerning the knowledge of the mothers regarding cardiac problems, most of them showed poor knowledge (87.2\%), while only $12.8 \%$ reported fair/good knowledge. Poor knowledge was more significantly reported among mothers of congenital cases $(91.9 \%)$, compared to $69.2 \%$ among mothers of RF/RHD cases. $\left(\chi^{2}=14.17, p<0.001\right)$

As regards the attitude of the mothers towards cardiac problems, about half of the sample (46.0\%) showed negative attitude, a figure which was significantly higher among mothers of congenital cases (54.7\%), compared to mothers of RF/RHD cases $(12.8 \%)$, the majority of mothers of $\mathrm{RF} / \mathrm{RHD}$ cases (87.2\%) showed positive attitude towards the disease $\left(\chi^{2}=21.82, p<0.00\right)$.

Concerning mother reaction after diagnosis, the most recorded reaction was acceptance $(41.2 \%)$, followed by sadness (33.2\%), shock (16.0\%), and deny (9.6\%). Shock and sadness were significantly more 
common among mothers of congenital cases ( $16.9 \%$ and $37.2 \%$, respectively)), compared to mothers of $\mathrm{RF} / \mathrm{RHD}$ cases $(12.8 \%$ and 17.95, respectively), while deny and acceptance were significantly more common among mothers of $\mathrm{RF} / \mathrm{RHD}$ cases $(17.9 \%$ and $51.4 \%$, respectively), compared to mothers of congenital cases $(7.4 \%$ and $38.5 \%$, respectively)

Table 2: shows the percentage distribution of mothers of children with cardiac problems according to their use of different coping methods. The results revealed that "Pray; trust in god" coping method was always used by most of mothers of children with cardiac problems $(92.5 \%)$, followed by "Accept the situation as it is" (88.0\%), and "Hope that things will be better" (73.5\%). More than half of the mothers $(55.5 \%)$ reported that they always "Resign themselves to the situation because it is their fate" while $28.0 \%$ reported that they always "Worry".

On the other hand, the least used coping methods by the mothers were "Take drugs (hypnotics, sedatives), where only $1 \%$ variables. reported using this coping method, followed by "Eat; chew gum" where only $6 \%$ reported using this method, "Blame someone else for your problem", where only $8.5 \%$ reported using this method, "Let someone else solve the problem" and "Take tea, coffee, and/or cigarette" where $12.5 \%$ reported using these coping methods

Table 3 showed significant association between percent mean score of copying skills and the duration of illness $(p<0.01)$, previous hospitalization $(p=0.01)$ and The percent mean total score was significantly higher with longer duration of illness, with history of previous hospitalization, and with negative attitude of mothers to illness. As for the affective copying skills, significant association was evident for all these variables except for mothers' attitudes $(p=0.59)$, while problemoriented copying skills score was significantly associated only with mothers' attitudes. $(p<0.001)$. However, the table showed no significant association between the percent mean score of copying skills and any of other 
As regards the duration of illness, the study revealed that the percent mean affective, problem-oriented, and total scores of mothers increase with increasing duration of illness. The percent mean affective and total scores were significantly higher when the duration of illness of the children was 5-10 years (43.92 \pm 10.23 and $42.30 \pm 10.53$, respectively), compared to $36.27 \pm 11.15$ and $36.28 \pm 10.59$, respectively when the duration of illness $<5$ years $(p<0.05)$. Percent mean problem-oriented score was higher when the duration of illness $\geq 11$ years $(50.00 \pm 21.02)$ compared to $36.31 \pm 15.93$ when the duration of illness $<5$ years.

Mothers of children who reported previous hospitalization recorded higher percent mean affective, problem-oriented, and total scores (39.47 $\pm 10.45,38.48 \pm 15.66 \pm$, and $39.32 \pm 9.49$, respectively) compared to mothers of children who reported no previous hospitalization (35.71 $\pm 11.80,35.90 \pm 16.80$, and $35.77 \pm 11.37$, respectively). The difference was statistically significant concerning the affective and total scores $(p<0.05)$.
As regards the attitude of the mother towards the cardiac problems, the study revealed that the percent mean problemoriented and total scores of mothers were significantly higher with negative attitude of the mothers $(40.45 \pm 17.66$ and $38.69 \pm 11.63$, respectively) compared to positive attitude (34.06 \pm 14.57 and $36.08 \pm 9.81$, respectively) $(p<0.05)$

The present study revealed that the percent mean score of use of problemoriented coping methods $(37.00 \pm 16.34 \%)$ was nearly equal to that for affective-oriented coping methods (37.42 $\pm 11.40 \%)$.

Table 4 shows multiple regression analysis of copying skills among mothers of children with cardiac problems with some independent variables. Duration of illness, knowledge, and attitude of mothers regarding the cardiac problems were the only significant predictors of the coping skills. High percent mean total coping scores was more likely among mothers of children with longer disease duration and those whose mother showed negative attitude towards cardiac problems $(p<0.05)$. High 
percent mean affective scores was significant among mothers with fair/good knowledge and negative attitude $(p<0.05)$. High percent cardiac problems $(p<0.05)$. mean problem-oriented scores was more likely among mothers with negative attitude towards 
Table (1): Distribution of the studied sample by cardiac problems and some variables

\begin{tabular}{|c|c|c|c|c|c|c|}
\hline \multirow{3}{*}{ Variables } & \multicolumn{4}{|c|}{ Cardiac problems } & \multirow{2}{*}{\multicolumn{2}{|c|}{ Total }} \\
\hline & \multicolumn{2}{|c|}{ Congenital } & \multicolumn{2}{|c|}{ RF/RHD } & & \\
\hline & $\begin{array}{l}\text { No. } \\
148\end{array}$ & $\begin{array}{r}\% \\
74.0\end{array}$ & $\begin{array}{l}\text { No. } \\
39\end{array}$ & $\begin{array}{c}\% \\
26.0\end{array}$ & $\begin{array}{c}\text { No } \\
187\end{array}$ & $\begin{array}{c}\% \\
100\end{array}$ \\
\hline \multicolumn{7}{|l|}{ Age (years) } \\
\hline$-\quad<6$ & 124 & 83.7 & 1 & 2.5 & 125 & 66.8 \\
\hline$-6-$ & 18 & 12.2 & 18 & 46.2 & 36 & 19.3 \\
\hline$-12+$ & 6 & 4.1 & 20 & 51.3 & 26 & 13.9 \\
\hline$\chi^{2}, p$ & \multicolumn{6}{|c|}{$98.5,0.00^{*}$} \\
\hline Mean(SD) & \multicolumn{6}{|c|}{$5.47(5.21)$} \\
\hline \multirow{3}{*}{$\begin{array}{cl}\text { Sex } & \\
- & \text { Male } \\
- & \text { female }\end{array}$} & & & & & & \\
\hline & 87 & 58.8 & 21 & 53.8 & 108 & 57.8 \\
\hline & 61 & 41.2 & 18 & 46.2 & 79 & 42.2 \\
\hline & \multicolumn{6}{|c|}{$0.31,0.57$} \\
\hline Mother education & & & & & & \\
\hline$-\quad$ low & 69 & 46.6 & 23 & 59.0 & 92 & 49.2 \\
\hline - $\quad$ middle & 67 & 45.3 & 14 & 35.9 & 81 & 43.3 \\
\hline - high. & 12 & 8.1 & 2 & 5.1 & 14 & 7.5 \\
\hline$\chi^{2}, p$ & \multicolumn{6}{|c|}{$1.94,0.37$} \\
\hline \multirow{3}{*}{$\begin{array}{cc}\text { Mother work } \\
-\quad \text { working } \\
- & \text { not working } \\
\end{array}$} & & & \multirow{4}{*}{$\begin{array}{c}4 \\
35 \\
0.02\end{array}$} & & & \\
\hline & 14 & 9.5 & & 10.3 & 18 & 9.6 \\
\hline & 134 & 90.5 & & 89.7 & 169 & 90.4 \\
\hline$\chi^{2}, p$ & \multicolumn{5}{|c|}{$0.02,0.88$} & \\
\hline \multirow{4}{*}{$\begin{array}{cl}\text { Cardiac lesions } \\
- & \text { no lesion } \\
- & \text { single lesion } \\
- & \text { multiple lesions }\end{array}$} & & & \multirow{5}{*}{$\begin{array}{c}9 \\
20 \\
10 \\
38.8\end{array}$} & & & \\
\hline & - & - & & 23.1 & 9 & 4.8 \\
\hline & 121 & 81.8 & & 51.3 & 141 & 75.4 \\
\hline & 27 & 18.2 & & 25.6 & 37 & 19.8 \\
\hline$\chi^{2}, p$ & \multicolumn{5}{|c|}{$38.81,0.00^{*}$} & \\
\hline Age at diagnosis (years) & & & \multirow{4}{*}{$\begin{array}{c}6 \\
26 \\
7 \\
\end{array}$} & & & \\
\hline$-\quad<6$ & 140 & 94.6 & & 15.4 & 146 & 78.1 \\
\hline$-\quad 6-$ & 6 & 4.1 & & 66.7 & 32 & 17.1 \\
\hline$-\quad \geq 12$ & 2 & 1.3 & & 17.9 & 9 & 4.8 \\
\hline$\chi^{2}, p$ & \multicolumn{6}{|c|}{$113.18,0.00^{*}$} \\
\hline Median & & & & & & \\
\hline Duration of illness (years) & & & & & & \\
\hline$-\quad<5$ & 132 & 89.2 & 26 & 66.7 & 158 & 84.5 \\
\hline$-\quad 5-$ & 13 & 8.8 & 13 & 33.3 & 26 & 13.9 \\
\hline$-\quad \geq 11$ & 3 & 2.0 & - & 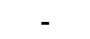 & 3 & 1.6 \\
\hline$\chi^{2}, p$ & & & 16.0 & & & \\
\hline Mean(SD) & & & & & & \\
\hline Previous hospitalization & & & & & & \\
\hline - yes & 62 & 41.9 & 18 & 6.2 & 80 & 42.8 \\
\hline$-\quad$ no & 86 & 58.1 & 21 & 3.8 & 107 & 57.2 \\
\hline$\chi^{2}, p$ & & & & & & \\
\hline
\end{tabular}


Table (1): Cont.

\begin{tabular}{|c|c|c|c|c|c|c|c|}
\hline $\begin{array}{c}\text { Knowledo } \\
- \\
-\end{array}$ & $\begin{array}{l}\text { Poor. } \\
\text { Pooir/good. }\end{array}$ & $\begin{array}{c}136 \\
12\end{array}$ & $\begin{array}{c}91.9 \\
8.1\end{array}$ & $\begin{array}{l}27 \\
12\end{array}$ & $\begin{array}{l}69.2 \\
30.8\end{array}$ & $\begin{array}{c}163 \\
24\end{array}$ & $\begin{array}{l}87.2 \\
12.8\end{array}$ \\
\hline$\chi^{2}, p$ & & \multicolumn{6}{|c|}{$14.17,<0.001^{*}$} \\
\hline $\begin{array}{c}\text { Attitude } \\
- \\
- \\
\end{array}$ & $\begin{array}{l}\text { Negative } \\
\text { positive }\end{array}$ & $\begin{array}{l}81 \\
67 \\
\end{array}$ & $\begin{array}{l}54.7 \\
45.3 \\
\end{array}$ & $\begin{array}{c}5 \\
34 \\
\end{array}$ & $\begin{array}{l}12.8 \\
87.2 \\
\end{array}$ & $\begin{array}{c}86 \\
101 \\
\end{array}$ & $\begin{array}{l}46.0 \\
54.0\end{array}$ \\
\hline$\chi^{2}, p$ & & \multicolumn{6}{|c|}{$21.82,<0.00^{*}$} \\
\hline $\begin{array}{c}\text { Mother re } \\
- \\
- \\
- \\
-\end{array}$ & $\begin{array}{l}\text { action } \\
\text { shock } \\
\text { sadness } \\
\text { deny } \\
\text { acceptance }\end{array}$ & $\begin{array}{l}25 \\
55 \\
11 \\
57\end{array}$ & $\begin{array}{c}16.9 \\
37.2 \\
7.4 \\
38.5\end{array}$ & $\begin{array}{c}5 \\
7 \\
7 \\
20\end{array}$ & $\begin{array}{l}12.8 \\
17.9 \\
17.9 \\
51.4\end{array}$ & $\begin{array}{l}30 \\
62 \\
18 \\
77\end{array}$ & $\begin{array}{c}16.0 \\
33.2 \\
9.6 \\
41.2\end{array}$ \\
\hline$\chi^{2}, p$ & & \multicolumn{6}{|c|}{$8.52,0.03^{*}$} \\
\hline
\end{tabular}

${ }^{*}$ Statistically significant

Table (2): Percentage distribution of mothers of children with cardiac problems according to their use of different coping methods

\begin{tabular}{|c|c|c|c|c|}
\hline \multirow[t]{3}{*}{ Coping Method } & \multirow{3}{*}{$\begin{array}{l}\text { Coping } \\
\text { Group }\end{array}$} & \multicolumn{3}{|c|}{ Response } \\
\hline & & Never & Sometimes & Always \\
\hline & & $(0)$ & $(1)$ & $(2)$ \\
\hline Hope that things will be better & A & 1.0 & 25.5 & 73.5 \\
\hline Pray; trust in god & A & 0.5 & 7.0 & 92.5 \\
\hline Accept the situation as it is & $\mathrm{P}$ & 2.5 & 9.5 & 88.0 \\
\hline Worry & A & 29.5 & 42.5 & 28.0 \\
\hline $\begin{array}{l}\text { Find out more about the situation so you can } \\
\text { handle it better }\end{array}$ & $P$ & 50.5 & 39.0 & 10.5 \\
\hline Get nervous & A & 48.0 & 33.0 & 19.0 \\
\hline $\begin{array}{l}\text { Drawn on past experience to help you handle the } \\
\text { situation }\end{array}$ & $\mathrm{P}$ & 54.0 & 40.0 & 6.0 \\
\hline Cry; get depressed & $\mathrm{A}$ & 48.5 & 43.5 & 8.0 \\
\hline $\begin{array}{l}\text { Try out different ways of solving the problem to see } \\
\text { which works best }\end{array}$ & $\mathrm{P}$ & 62.5 & 32.5 & 5.0 \\
\hline $\begin{array}{l}\text { Go to sleep figuring things will look better in the } \\
\text { morning }\end{array}$ & A & 46.5 & 39.0 & 14.5 \\
\hline Want to be alone & $\mathrm{A}$ & 53.0 & 35.5 & 11.5 \\
\hline Seek comfort or help from family or friends & $\mathrm{P}$ & 41.5 & 40.5 & 18.0 \\
\hline $\begin{array}{l}\text { Talk the problem over with someone who has been } \\
\text { in the same type of situation }\end{array}$ & $\mathrm{P}$ & 34.0 & 44.5 & 21.5 \\
\hline $\begin{array}{l}\text { Resign yourself to the situation because it is your } \\
\text { fate }\end{array}$ & $\mathrm{A}$ & 11.0 & 33.5 & 55.5 \\
\hline Try to put the problem out of your mind & A & 53.0 & 38.5 & 8.5 \\
\hline Blame someone else for your problems & $\mathrm{A}$ & 91.5 & 7.5 & 1.0 \\
\hline $\begin{array}{l}\text { Don't worry about it, everything probably works out } \\
\text { fine }\end{array}$ & A & 26.0 & 53.0 & 21.0 \\
\hline Eat; chew gum & $\mathrm{A}$ & 94.0 & 4.5 & 1.5 \\
\hline Let someone else solves the problem & $\mathrm{P}$ & 87.5 & 11.0 & 1.5 \\
\hline Take tea, coffee, and/or cigarette & A & 87.5 & 10.0 & 2.5 \\
\hline Take drugs (hypnotics, and/or sedatives) & A & 99.0 & 1.0 & - \\
\hline
\end{tabular}

$A=$ affective oriented

$\mathrm{P}=$ problem oriented 
Table (3): Distribution of mothers of children with cardiac problems according to the percent mean coping scores and some variables

\begin{tabular}{|c|c|c|c|}
\hline \multirow[t]{2}{*}{ Variable } & \multicolumn{3}{|c|}{ Percent mean(SD) coping score } \\
\hline & Affective-oriented & Problem-oriented & Total \\
\hline \begin{tabular}{cl}
\multicolumn{2}{l}{ Age } \\
$-\quad<6$ \\
$-\quad 6-$ \\
$-\quad 12+$
\end{tabular} & $\begin{array}{c}36.59(11.21) \\
38.43(13.21) \\
39.39(9.36)\end{array}$ & $\begin{array}{l}35.99(16.09) \\
36.56(15.39) \\
41.51(18.18)\end{array}$ & $\begin{array}{c}36.39(10.46) \\
37.81(12.18) \\
40.10(9.52)\end{array}$ \\
\hline$p$-value & 0.38 & 0.23 & 0.21 \\
\hline $\begin{array}{cl}\text { Sex } & \\
- & \text { male } \\
- & \text { female. }\end{array}$ & $\begin{array}{l}37.14(11.92) \\
37.81(10.70)\end{array}$ & $\begin{array}{l}36.64(16.99) \\
37.47(15.50)\end{array}$ & $\begin{array}{c}36.97(11.72) \\
37.70(9.29)\end{array}$ \\
\hline$p$-value & 0.69 & 0.54 & 0.53 \\
\hline $\begin{array}{ll}\text { Birth order } \\
-\quad \text { first. } \\
-\quad \text { second. } \\
-\quad \text { third or more. }\end{array}$ & $\begin{array}{l}37.16(10.11) \\
37.09(11.79) \\
38.04(12.04) \\
\end{array}$ & $\begin{array}{l}36.37(16.37) \\
35.71(15.66) \\
39.06(17.14) \\
\end{array}$ & $\begin{array}{l}36.90(10.19) \\
36.63(10.50) \\
38.38(11.51) \\
\end{array}$ \\
\hline$p$-value & 0.87 & 0.44 & 0.59 \\
\hline \begin{tabular}{ll}
\multicolumn{2}{l}{ Mother education } \\
- & low \\
- & middle \\
- & high (university or \\
& more) \\
\end{tabular} & $\begin{array}{c}37.92(11.88) \\
36.18(9.75) \\
41.32(16.01)\end{array}$ & $\begin{array}{l}35.43(16.66) \\
38.43(15.27) \\
39.79(19.95)\end{array}$ & $\begin{array}{c}37.09(10.90) \\
36.93(9.95) \\
40.81(13.94)\end{array}$ \\
\hline$p$-value & 0.24 & 0.37 & 0.44 \\
\hline \begin{tabular}{ll}
\multicolumn{1}{l}{ Mother work } \\
$-\quad$ working \\
$-\quad$ not working
\end{tabular} & $\begin{array}{l}35.90(12.90) \\
37.58(11.25)\end{array}$ & $\begin{array}{l}42.48(14.57) \\
36.42(16.44)\end{array}$ & $\begin{array}{l}38.09(11.16) \\
37.20(10.72)\end{array}$ \\
\hline$p$ - value & 0.56 & 0.10 & 0.76 \\
\hline $\begin{array}{ll}\text { Family size } \\
-\quad \leq 3 \\
-\quad 4- \\
-\quad \geq 6\end{array}$ & $\begin{array}{l}36.01(10.00) \\
37.73(11.96) \\
37.74(10.86)\end{array}$ & $\begin{array}{l}37.73(16.93) \\
37.06(16.48) \\
36.29(15.64)\end{array}$ & $\begin{array}{c}36.50(10.54) \\
37.51(11.18) \\
37.25(9.54)\end{array}$ \\
\hline$p$-value & 0.72 & 0.95 & 0.89 \\
\hline \begin{tabular}{cc}
\multicolumn{2}{l}{ Diagnosis } \\
$-\quad$ Congenital \\
$-\quad$ rheumatic \\
\end{tabular} & $\begin{array}{c}37.57(11.80) \\
38.73(9.28)\end{array}$ & $\begin{array}{c}36.53(16.40) \\
37.17(15.30)\end{array}$ & $\begin{array}{c}37.22(11.10) \\
38.21(9.04)\end{array}$ \\
\hline$p$-value & 0.31 & 0.86 & 0.31 \\
\hline $\begin{array}{l}\text { Age at diagnosis (years) } \\
-\quad<6 \\
-\quad 6- \\
-\quad \geq 12\end{array}$ & $\begin{array}{c}37.35(11.63) \\
37.25(11.27) \\
38.96(8.95)\end{array}$ & $\begin{array}{l}36.56(16.27) \\
36.67(16.50) \\
44.15(16.54)\end{array}$ & $\begin{array}{l}37.09(10.76) \\
37.06(10.71) \\
40.69(10.88)\end{array}$ \\
\hline$p$-value & 0.90 & 0.33 & 0.56 \\
\hline 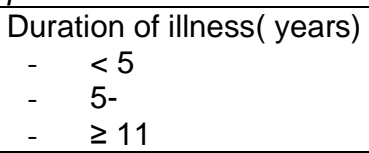 & $\begin{array}{l}36.27(11.15) \\
43.92(10.23) \\
36.60(16.33)\end{array}$ & $\begin{array}{l}36.31(15.93) \\
39.04(17.66) \\
50.00(21.02)\end{array}$ & $\begin{array}{c}36.28(10.59) \\
42.30(10.53) \\
41.07(9.19)\end{array}$ \\
\hline$p$-value & $<0.001^{*}$ & 0.19 & $0.01^{*}$ \\
\hline
\end{tabular}


Table (3): Cont.

\begin{tabular}{|c|c|c|c|}
\hline $\begin{array}{l}\text { Previous hospitalization } \\
-\quad \text { yes } \\
-\quad \text { no }\end{array}$ & $\begin{array}{l}39.74(10.45) \\
35.71(11.80)\end{array}$ & $\begin{array}{l}38.48(15.66) \\
35.90(16.80)\end{array}$ & $\begin{array}{c}39.32(9.49) \\
35.77(11.37)\end{array}$ \\
\hline$p$-value & $0.01^{*}$ & 0.21 & $0.01^{*}$ \\
\hline $\begin{array}{cc}\text { Scholastic achievement } \\
-\quad \text { weak/fair } \\
-\quad \text { good/VG }\end{array}$ & $\begin{array}{l}42.20(10.45) \\
38.24(11.93)\end{array}$ & $\begin{array}{l}42.85(19.94) \\
38.13(16.04)\end{array}$ & $\begin{array}{c}42.42(8.63) \\
38.21(11.48)\end{array}$ \\
\hline$p$-value & 0.12 & 0.55 & 0.17 \\
\hline $\begin{aligned} & \text { Knowledge } \\
&- \text { Poor. } \\
&- \text { Fair/good. }\end{aligned}$ & $\begin{array}{l}37.62(11.18) \\
39.14(12.30)\end{array}$ & $\begin{array}{l}36.46(15.94) \\
38.10(17.71)\end{array}$ & $\begin{array}{l}37.23(10.52) \\
38.79(11.93)\end{array}$ \\
\hline$p$-value & 0.57 & 0.67 & 0.51 \\
\hline $\begin{array}{cl}\text { Attitude } & \\
- & \text { Negative } \\
- & \text { positive }\end{array}$ & $\begin{array}{l}37.81(11.98) \\
37.10(10.92)\end{array}$ & $\begin{array}{l}40.45(17.66) \\
34.06(14.57)\end{array}$ & $\begin{array}{c}38.69(11.63) \\
36.08(9.81)\end{array}$ \\
\hline$p$-value & 0.59 & $<0.001^{*}$ & $<0.05$ \\
\hline
\end{tabular}

* Statistically significant

Percent mean (SD) affective coping score $=37.42(11.40)$

Percent mean (SD) problem-oriented coping score $=37.00(16.34)$

Percent mean (SD) total coping score $=37.28(10.73)$

Table (4): Multiple regression analysis of copying skills among mothers of children with cardiac problems with some independent variables

\begin{tabular}{|l|l|l|l|}
\hline \multirow{2}{*}{\multicolumn{1}{|c|}{ Variables }} & \multicolumn{3}{c|}{ Copy strategies (score \%) } \\
\cline { 2 - 4 } & affective & problem & total \\
\cline { 2 - 4 } & $\beta(\mathrm{SE})$ & $\beta(\mathrm{SE})$ & $\beta(\mathrm{SE})$ \\
\hline Age at diagnosis & $0.13(0.32)$ & $-0.16(0.46)$ & $0.03(.31)$ \\
\hline Previous hospitalization (yes=1) & $2.54(1.68)$ & $-0.05(2.41)$ & $1.68(1.60)$ \\
\hline Duration of illness & $0.59(0.31)$ & $0.76(0.45)$ & $0.65(0.30)^{*}$ \\
\hline Diagnosis (Rheumatic=1) & $-0.26(3.21)$ & $5.66(4.62)$ & $1.71(3.06)$ \\
\hline Knowledge (score) & $0.09(0.04)^{*}$ & $-0.11(0.06)$ & $0.03(0.04)$ \\
\hline Attitude (score) & $-1.03(0.5)^{*}$ & $-2.32(0.72)^{*}$ & $-1.46(0.47)^{*}$ \\
\hline
\end{tabular}

*.... Statistically significant

$\beta$... coefficient of determination

SE ... Standard error. 


\section{DISCUSSION}

The presence of a chronic illness in a child commits the family to long-term difficulties and problems comprising a variety of different stressors. Effective coping alleviates the problem and reduces emotional distress. If coping is ineffective, distress remains unchanged or becomes aggravated. ${ }^{12}$ How parents cognitively appraise concerns related to the care of their child will determine their coping strategies. ${ }^{13}$

The aim of the present work was to assess coping strategies of mothers of children with cardiac problems which are important for planning intervention programs to enhance their coping. In consistent with the study done by Yousef et al., (2004) $)^{14}$ on the mothers of mentally handicapped children, the present study revealed that "Pray; trust in god" coping method was always used by most of mothers of children with cardiac problems, followed by "Accept the situation as it is". These two coping methods are adopted by mothers in an attempt to maintain some control over the situation that facilitate effective adaptation and diminish a feeling of helplessness and hopelessness. ${ }^{15}$ It is well known that people in our community always pray and trust god when they face their problems to help them overcome the stress evoked by that problem. Also, religion gives them strength that helps them accept the situation. The third adopted coping method was "Hope that things will be better". Hopeful perspective is of importance for adaptation because it allows a person to become aware of alternative options for coping with stress. ${ }^{10}$ More than half of the mothers reported that they always "Resign themselves to the situation because it is their fate". This is because most people in our community believe that everything they face is their fate. However, about a quarter of the sample reported that they always "Worry."

Meanwhile, the least used coping methods by the mothers were "Take drugs (hypnotics, or sedatives), followed by "Eat; chew gum", "Blame someone else for your problem", "Let someone else solves the problem" and "Take tea, coffee, and/or cigarette". Taking drugs, chew gum, and take 
tea and coffee, and cigarette are not coping methods commonly used by females in our culture to help them face their problems, especially females of low socioeconomic class that constitute the study sample. Also, females belonging to this stratum depend usually upon themselves in solving their problems and never blame someone else.

Most people use multiple coping behaviors in any given stressful situation. The choice of behaviors is directed by the changing environment and the altering situation. ${ }^{16}$ The present study revealed that the percent mean score of use of problemoriented coping method $(37.00 \pm 16.34 \%)$ was nearly equal to that for affective-oriented coping method (37.42 $\pm 11.40 \%)$. Many studies indicated the need for balance between problem-oriented and affective-oriented coping methods. ${ }^{10,17}$ Others consider problem solving a pre requisite for accommodation and successful dealing with the problems. ${ }^{15,18}$

An individual's perception of the event is a critical factor in determining whether the event will be stressful or not. Parents who defined having a child with a disability in a positive way had more successful adaptations and family strengths. ${ }^{15}$ The present work revealed that about half of the mothers showed negative attitude towards the cardiac problems, a figure which was significantly higher among mothers of congenital cases. Parents of children with $\mathrm{CHD}$ have identified psychological stress as their most significant problem ${ }^{19}$. Diagnostic procedures, medical and surgical interventions, perception of the child as "different," social problems, sports restrictions, uncertainty, and disease prognosis have also been identified as sources of stress ${ }^{20-22}$. Among parents of adolescents and young adults, a 1997 study reported that issues related to the perception of their offspring as different persisted. Additional concerns were related to decisions about disclosure of the diagnosis and transitioning offspring to self-management of the chronic illness. ${ }^{4}$

The present study revealed that the \% mean problem-oriented and total coping scores of mothers were significantly higher 
with negative attitude of the mothers. After adjusting for other variables, attitude of the mother was a significant predictor of coping including affective-, problem-oriented, and total scores. Negative attitude increases the psychological distress of the mother due to overprotection and more effort in caring for her child together with the sense of guilt and family problems that might occur. Therefore, she tries to increase her coping strategies to overcome this stress and solve the problem.

Despite recent advances in medical and surgical management of pediatric cardiac patients, the findings of the present study showed that mothers have important knowledge gaps, which were more significantly reported among mothers of congenital cases. Given the important role of parents in imparting knowledge of chronic illness to their child, the disappointing findings from previous studies reflect inadequacies of parental knowledge.23,24 After adjusting for other variables, high percent mean affective scores was significant among mothers with fair/good knowledge. The more information parents have, the less frightening the present and future will seem. Knowledge is empowering. It can help feel more in control of, and less a hostage to, the condition you must face. Information will also help the mother guide her child through the potentially complicated medical care system. ${ }^{23}$

Several researchers have hypothesized that parents are apt to follow similar patterns or predictable stages that most parents go through. Some examples of these stages include initial shock, denial, emotional disorganization, and reaching a final stage of adjustment or acceptance..$^{22,25}$ In the present study, the most recorded reaction was acceptance, followed by sadness, shock, and deny. Shock and sadness were significantly more common among mothers of congenital cases, while deny and acceptance were significantly more common among mothers of RF/RHD cases.

As regards the duration of illness, the present study revealed that the percent mean affective-, problem-oriented, and total scores of mothers increase with increasing duration of 
illness. After adjusting for other variables, duration of illness was a significant predictor of total coping strategies. The longer the duration of illness, the more will be the accommodation and experience in dealing with the problem. Most children with CHD are diagnosed in the perinatal period or in early infancy and thus; families must concurrently deal with the stress of a normative transition (new family member) and the stress of the diagnosis of a chronic illness. Caregiving demands are very high, especially in the early stages of illness ${ }^{22}$. Caregiving issues identified in the literature include difficulties with nutrition and feedings and intensive medication regimens ${ }^{21}$. However, mothers of children with CHD - in the present study - were not significantly different from those of children with RF/RHD in terms of coping strategy inspite of the significally longer duration of the former group.

Mothers of children who reported previous hospitalization in the present study, recorded higher percent mean affective-, problemoriented, and total coping strategy scores in the bivariate analysis. Hospitalized patients are the severe cases that may need surgery and more care from the mother. This may increase the stress and coping strategies of the mother. Despite the difficulties including hospitalization, however, many families adapt successfully22. After adjusting for all other variables in the present study, previous hospitalization was not a significant predictor of coping strategies.

\section{CONCLUSION:}

1. Mothers of children with cardiac problem have important knowledge gaps and show negative attitude towards their diseased children. Poor knowledge of and negative attitudes towards cardiac problems were significantly more prevalent among mothers of children with $\mathrm{CHD}$ than among those of children with RHD.

2. Coping strategies of mothers of children with cardiac problem are inadequate. However, high coping strategy scores are significantly associated with the longer duration of illness and the negative attitude of mothers, possibly 
due to prolonged exposure to stress and anxiety, and the feeling that their children are different. The type of cardiac defect is not a significant predictor of coping skills.

\section{RECOMMENDATIONS:}

In order to cope, families may benefit from communication with others who share common concerns, interests, and goals. Some factors that impact a family's ability to cope are: ability to understand/comprehend medical concepts, what the child's physical condition is and parent' perception that they "did something wrong" and caused the heart defect.

Support group for parents is a highly recommended strategy to help a family cope in different ways; provide emotional support for short-term and long-term coping, decrease feelings of isolation for those whose family and friends are at a loss on how to provide support, reduce anxiety and stress of family members, gather and share information about the same or similar defect, and focus on positive aspects of the situation.

\section{REFERENCES}

1. Refat M, Rashad E, El-Gazar FA, Shafie AM, Abou El-Nour MM, ElSherbini A, et al. A clinicoepidemiologic study of heart disease in school children in Menoufia, Egypt. Ann Saudi Med. 1994; 14(3): 225-9.

2. Bassili A, Zaher SR, Zaki A, AbdelFattah M, Tognoni $G$. Profile of secondary prophylaxis among children with rheumatic heart disease in Alexandria, Egypt. Eastern Mediterranean Health Journal. 2000; 6(2/3): 437-46.

3. Davis CC, Brown RT, Bakeman R, Campbell R. Psychological adaptation and adjustment of mothers of children with congenital heart diseases: stress, coping, and family functioning. Journal of Pediatric Psychology.1998; 23(4):219-28.

4. Sparacino PSA, Tong EM, Messias DKH, Foote D, Chesla CA, Gillis CL. The dilemmas of parents of adolescents and young adults with congenital heart disease. Heart \& Lung. 1997; 26(3): 187-95.

5. Abolfotouh MA, Tayel KY, Ayoub AI, ElSebaiei M. Preventive and clinical aspects of cardiac problems among school children in Alexandria. The Bulletin of High Institute of Public Health. 1994; 24: 735-47.

6. Major DA. Utilizing role theory to help employed parents cope with children's chronic illness. Health Education Research. 2003; 18(1):45-57.

7. Perez LM. Children coping with chronic illness. Paper presented at the annual meeting of the Southwest Education Research Association. Austin, TX, January 23, 1997. [Cited 2007 May 14]. Available from:

http://www.eric.ed.gov/ERICDocs/data/e ricdocs2sql.

8. Folkman S, Lazarus RS. An analysis of coping in a middle-aged community sample. Journal of Health and Social Behavior. 1980; 21: 219-39. 
9. Carver CS, Scheier MF, Weintraub JK. Assessing coping strategies: A theoretically based approach. Journal of Personality and Social Psychology. 1989; 56: 267-83.

10. Jalowiec A, Powers M. Stress and coping in hypertensive and emergency room patients. Journal of Personality and Social Psychology. 1981; 37(1):111.

11. Kanona AM. The relationship between the perceived stressors and coping strategies among arthritic patients in Shebin El-Kom University Hospital. Unpublished DNS, Faculty of Nursing, Menoufiya University. 2002.

12. Tansella CZ. Psychosocial factors and chronic illness in childhood. Eur Psychiatry. 1995; 10:297-305.

13. Heaman DJ. Perceived stressors and coping Strategies of parents who have children with developmental disabilities: A comparison of mothers with fathers. Journal of Pediatric Nursing. 1995; 10(5): 311-20.

14. Yousef Y, Darwish I, Shehata A. Behavioral problems of mentally handicapped children encountered by their mothers. Bulletin of High Institute of Public Health. 2004; 34(1): 1-22.

15. Melnyk BM, Moldenhouer Z, Fienstien NF, Small L. Coping in parents of children who are chronically ill: strategies for assessment and intervention. Pediatric Nursing. 2001; 27(6): 547-57.

16. Chien-yun W, Yan-Yao L, Karin B, Oraanong $W$. Coping behaviors of individuals with chronic obstructive pulmonary disease. MedSurg Nursing. 2001. [Cited 2007 May 20]. Available from: http://findarticles.com/p/articles /mi_m0FSS/is_6_10/ai_n18612800>HT ML.

17. Eakes G. Chronic sorrow: the lived experience of parents of chronically ill children. Archives of Psychiatric Nursing. 1995; 9: 77-84.

18. Baldaia L. An educational approach to successful management of childhood asthma as a chronic illness. Journal of Pediatric Nursing. 1996; 11: 335-46.

19. Samanek M. Congenital heart malformations: Prevalence, severity, survival, and quality of life. Cardiology in the Young. 2000; 10: 179-85.

20. Gudmundsdottir M, Gillis CL, Sparacino PSA, Tong EM, Messias DKH, Foote D. Congenital heart defects and parentadolescent coping. Families, Systems, \& Health.1996; 14(2): 245-55.

21. Morelius $\mathrm{E}$, Lundh $\mathrm{U}$, Nelson $\mathrm{N}$. Parental stress in relation to the severity of congenital heart disease in offspring. Pediatric Nursing. 2002; 28(1): 28-32.

22. Tak YR, McCubbin M. Family stress, perceived social support, and coping following the diagnosis of a child's congenital heart disease. Journal of Advanced Nursing. 2002; 39(3): 190-8.

23. Cheuk DK, Wong SM, Choi YP, Chau AK, Cheung YF. Parents' understanding of their child's congenital heart diseases. Heart. 2004; 90:435-9.

24. Cetta F, Bell TJ, Podlecki DD. Parental knowledge of bacterial endocarditis prophylaxis. Pediatr Cardiol. 1993; 14:220-2.

25. Blacher J. Sequential stages of parental adjustment to the birth of a child with handicaps: Fact or artifact? Mental Retardation. 1984; 22(2) 55-68. 